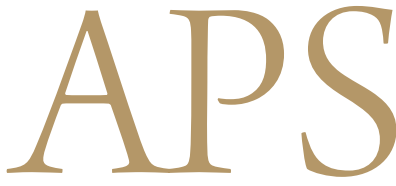

Archives of Plastic Surgery

\title{
A 20-year experience of immediate mandibular reconstruction using free fibula osteocutaneous flaps following ameloblastoma resection: Radical resection, outcomes, and recurrence
}

\author{
Koh Siang Chai ${ }^{1}$, Farah Hany Omar ${ }^{1}$, Arman Zaharil Mat Saad ${ }^{1,2}$, Wan Azman Wan Sulaiman ${ }^{1}$, \\ Ahmad Sukari Halim ${ }^{1}$ \\ ${ }^{1}$ Reconstructive Sciences Unit, School of Medical Sciences, Universiti Sains Malaysia, Kota Bharu; ${ }^{2}$ Plastic and Reconstructive Surgery Unit, \\ Management \& Science University Medical Centre, Shah Alam, Malaysia
}

Background The mandible is an important structure that is located in the lower third of the face. Large mandibular defects after tumor resection cause loss of its function. This study assessed the outcomes and tumor recurrence after immediate mandibular reconstruction using a free fibula osteocutaneous flap following radical resection of ameloblastoma.

Methods This is a retrospective non-randomized study of outcomes and tumor recurrence of all patients diagnosed with mandibular ameloblastoma from August 1997 until August 2017 (20 years) requiring free fibula osteocutaneous flap reconstruction at a single institution. The patients were identified through an electronic operative database; subsequently, their medical records and photo documentation were retrieved.

Results Twenty-seven patients were included in this study. Eighteen patients were male, while nine were female. The majority of the patients (48.1\%) were in their third decade of life when they were diagnosed with ameloblastoma. All of them underwent radical resection of the tumor with a surgical margin of $2 \mathrm{~cm}$ (hemimandibulectomy in cases with a large tumor) and immediate mandibular reconstruction with a free fibula osteocutaneous flap. Two patients required revision of a vascular anastomosis due to venous thrombosis postoperatively, while one patient developed a flap recipient site infection. The flap success rate was 100\%. There was no tumor recurrence during a mean follow-up period of 5.6 years.

Conclusions Mandibular ameloblastoma should be treated with segmental mandibulectomy (with a surgical margin of $2 \mathrm{~cm}$ ) to reduce the risk of recurrence. Subsequent mandibular and adjacent soft tissue defects should be reconstructed immediately with a free fibula osteocutaneous flap.

Keywords Ameloblastoma / Mandibular reconstruction / Free tissue flaps
Correspondence:

Arman Zaharil Mat Saad Plastic and Reconstructive Surgery Unit, Management \& Science University Medical Centre, Jalan Boling Padang 13/64, Seksyen 13, Shah Alam 40100, Malaysia Tel: +60-3-5526-2888 Fax: +60-3-5524-3656 E-mail:armanzaharil@gmail.com

This article was presented at the 16th International Congress of OSAPS in conjunction with the ISAPS Symposium, and the 10th National Congress and 22nd Annual Scientific Meeting of InaPRAS, on July 21, 2018, in Bali, Indonesia. 


\section{INTRODUCTION}

Ameloblastoma is a rare, slowly growing, locally invasive odontogenic tumor of epithelial origin that involves the mandible in $80 \%$ of cases and occurs mostly in individuals aged $30-60$ years old. Although many conservative surgical treatments, such as enucleation, cauterization, and curettage have been suggested, owing to the high risk of recurrence, radical surgical treatment with a wide margin remains the choice of treatment for many surgeons $[1,2]$.

The mandible is an important structure that is located in the lower third of the face. It provides a platform to house the dentition for mastication, facilitates speech and swallowing, and maintains adequate space in the oral cavity for the tongue to function normally [3].

Large defects in the mandible due to radical tumor resection could cause impairment of all these functions, as well as social isolation due to an asymmetrical or abnormal projection of the lower third of the face. Therefore, mandibular reconstruction is vital to restore all these functions and to provide satisfactory aesthetic outcomes for individuals to reintegrate into society [4]. The free fibula osteocutaneous flap fulfills these requirements by providing high cortical bone content, which favors long-term dental rehabilitation, as well as adequate soft tissue coverage for both extra- and intra-oral defects.

A systematic review of mandibular reconstruction with vascularized bone flaps over 25 years by Brown et al. [5] revealed that, although many authors have published their results of mandibular reconstruction, the outcomes reported lack consistency that would enable a reliable comparison between different studies. In an effort to solve these issues, Brown et al. [5] suggested that future publications should be standardized to include patients' dental status, classification, length of defects, method of reconstruction, number of osteotomies, early and late complications, and the final outcomes of reconstruction.

This single-center study was conducted to report our experiences and outcomes of radical surgical treatment of ameloblastoma, followed by immediate mandibular reconstruction using a free fibula osteocutaneous flap, in an Asian population. Compared to other studies, our patients had different clinical circumstances, such as a tendency for late presentation with huge tumors due to neglect and a tendency to seek traditional treatment first. In an attempt to enable a reliable comparison of our outcomes with other studies, we have done our best to report our results according to the requirements suggested by Brown et al. [5].

\section{METHODS}

\section{Patients}

This is a retrospective, non-randomized study of outcomes and recurrence in all patients diagnosed with mandibular ameloblastoma from August 1997 to August 2017 who underwent ameloblastoma resection followed by immediate free fibula osteocutaneous flap reconstruction at our institution. The patients were identified through an electronic operative database; subsequently, their medical records and photo documentation were retrieved. Photographic consent and release permission were also obtained from patients. Patients with medical records that were no longer available were excluded from this study. This study was exempted by our Institutional Review Board (IRB) from ethical review (USM/JEPeM/18100480).

\section{Surgical techniques}

Mandibular resection was performed in several ways; when possible, segmental mandibulectomy with a surgical margin of $2 \mathrm{~cm}$ from the radiographic border of the tumor was performed, while in large tumors located around the ramus, hemimandibulectomy was performed. Immediate mandibular reconstruction with a free fibula osteocutaneous flap was performed following resection (Fig. 1). The maximum possible length of fibula bone

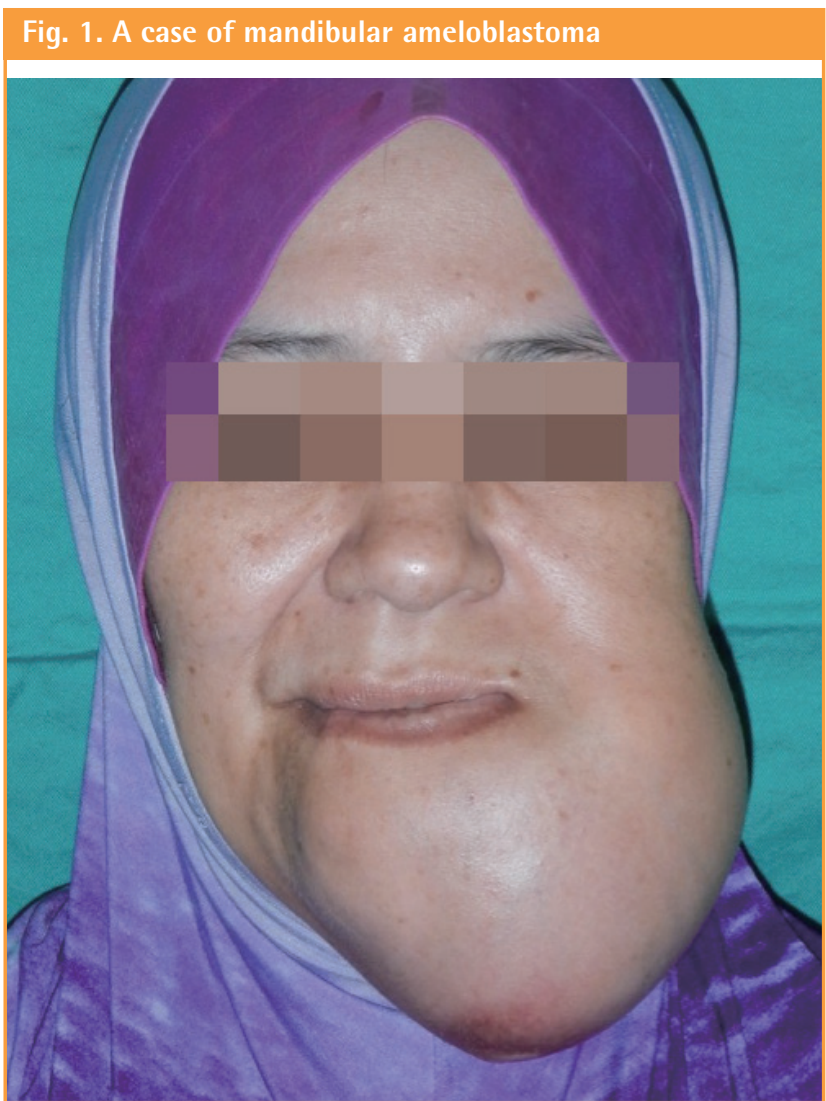


Fig. 2. Intraoperative and postoperative photographs

The patient underwent immediate mandibular reconstruction using free fibula osteocutaneous flap after ameloblastoma resection. (A, B) A free fibula osteocutaneous flap. (C) The harvested fibula flap was osteotomized into several segments to fit the contour of the titanium plate. (D) Flap inset. (E) One year after surgery.
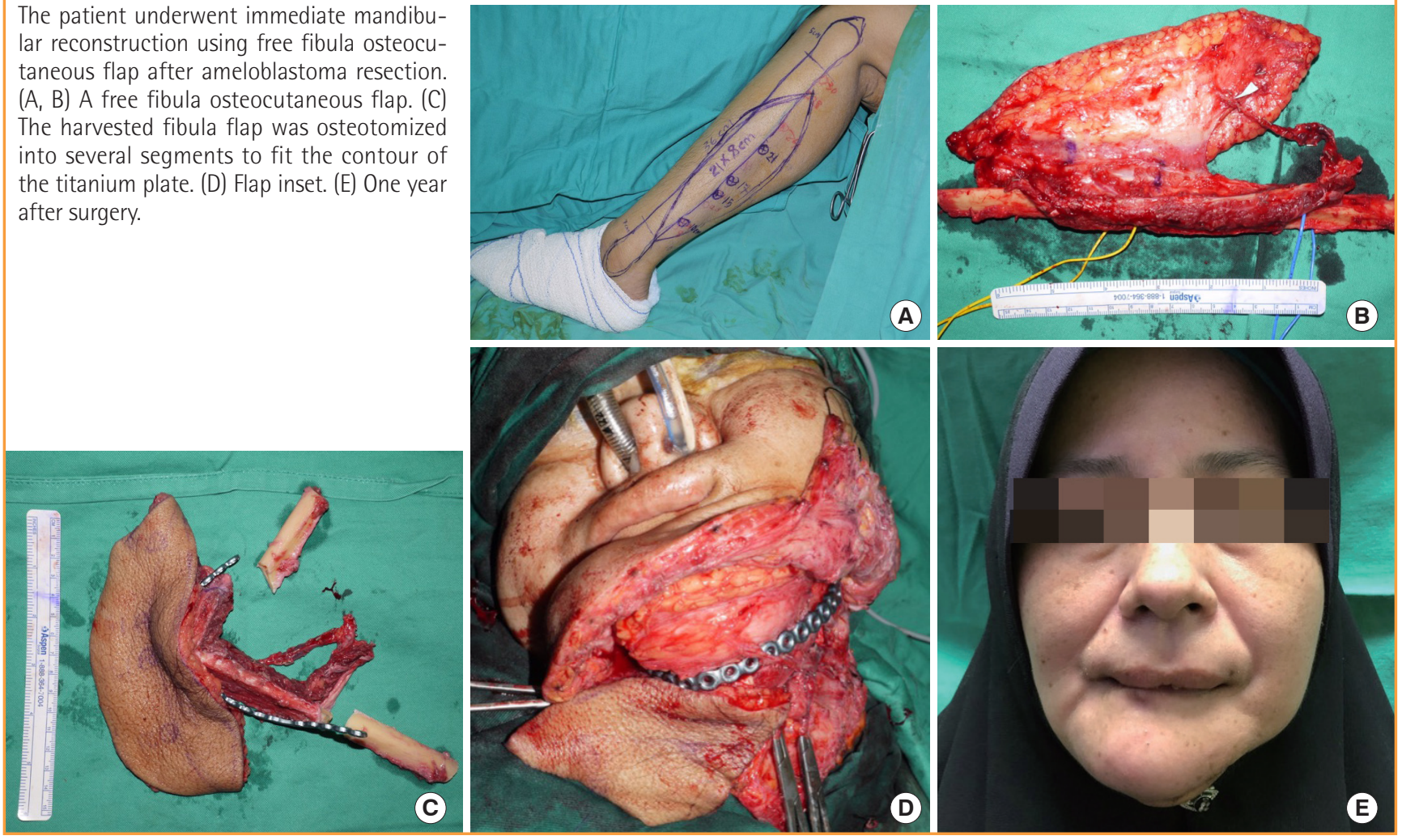

was harvested, leaving 5-7 cm of bone distally and proximally and preserving at least one perforator to the skin. The bone was osteotomized into two or three segments, which would be attached to a premolded mandibular reconstruction plate (OsteoMed, Dallas, TX, USA) with screws after dividing the donor artery and vein. The plate was placed at the lower border of the mandible to provide external definition of the jaw. A skin paddle was used to cover any adjacent extra- or intra-oral defect. If the adjacent defect was so small that a skin paddle was not required for coverage, the skin paddle was used as a platform for flap monitoring. In later cases, three-dimensional modeling was used to mold the plate. The harvested fibula was osteotomized into several segments to fit the contour of the titanium plate. The fibula flap was then secured with screws onto the titanium plate and inset onto the recipient site (Fig. 2). The peroneal vessels were anastomosed with adjacent vessels at the recipient site. No interposition vein grafts were required in this series. A skin paddle was used to cover intra- or extra-oral defects if needed.

The flap donor site was then assessed for primary closure. If primary closure would result in significant tension to the wound, the donor site wound was dressed with a modern dressing material. Delayed primary closure or split-thickness skin grafting was done 2 weeks later when soft tissue swelling had subsided and wound contraction had occurred.

\section{RESULTS}

A total of 27 patients were included in this study, of whom 18 (66.7\%) were men and nine $(33.3 \%)$ were women.

The majority of the patients were in their third decade of life when they were diagnosed with ameloblastoma $(n=13,48.1 \%)$. Three patients (11.1\%) were in their second decade of life, five patients (18.5\%) were in their fourth decade of life, four patients (14.8\%) were in their fifth decade of life, and two other patients (7.4\%) were in their sixth decade of life. Their mean age was 29.7 years.

In this series, ameloblastoma was mostly located in the lateral aspect (body or ramus) of the mandible, rather than the anterior aspect, and presented with an almost equal distribution between the left and right sides of the mandible (Table 1) [6]. The average dimensions of the tumors at presentation were $6.4 \mathrm{~cm}$ (length) $\times$ $4.9 \mathrm{~cm}$ (width) (length, 3.3-10.2 cm; width, 1.0-9.1 cm).

Patients' total fibula length ranged from 32 to $42 \mathrm{~cm}$. The harvested mean fibula length was $22.6 \mathrm{~cm}$, of which only $13.1 \mathrm{~cm}$ was used for reconstruction (Table 2).

The artery of the flap was anastomosed end-to-end to the recipient site artery in 26 cases (96.3\%), while end-to-side anastomosis was done in one case (3.7\%). The recipient arteries were the superior thyroid artery $(n=3,11.1 \%)$, facial artery $(n=9$, 
Table 1. Summary of epidemiological patterns and outcomes of surgery $(n=27)$

\begin{tabular}{|lc|}
\hline Parameter & No. (\%) \\
\hline Age group (yr) & \\
$10-19$ & $3(11.1)$ \\
$20-29$ & $13(48.1)$ \\
$30-39$ & $5(18.5)$ \\
$40-49$ & $4(14.8)$ \\
$50-59$ & $2(7.4)$ \\
Sex & \\
Male & $18(66.7)$ \\
Female & $9(33.3)$ \\
Tumor location & \\
Anterior & $3(11.1)$ \\
Left & $13(48.1)$ \\
Right & $11(40.8)$ \\
Presenting symptoms & \\
Mandibular swelling & $18(66.7)$ \\
Pain/discomfort & $7(25.9)$ \\
Infection & $2(7.4)$ \\
Mandibular defecta) & \\
Hemimandibulectomy (H) & $3(11.1)$ \\
Central (C) & $3(11.1)$ \\
Lateral defect-sparing condyle (L) & $21(77.8)$ \\
Pathologic pattern & \\
Solid/multicystic & $24(88.9)$ \\
Unicystic & $3(11.1)$ \\
Recurrence & 0 \\
Flap survival & $27(100)$ \\
Flap donor site closure & \\
Primary closure & $10(37)$ \\
Split-thickness skin grafting & $17(63)$ \\
\hline a)Classification of mandibular defects according to Jewer et al. [6]. \\
\end{tabular}

$33.3 \%)$, lingual artery $(n=1,3.7 \%)$, and superficial temporal artery $(\mathrm{n}=1,3.7 \%)$; however, in 13 cases $(48.1 \%)$, the recipient artery was not stated. Veins were anastomosed end-to-end in 25 cases $(92.6 \%)$, while end-to-side anastomosis were done in the two other cases (7.4\%). The recipient veins were the internal jugular vein $(n=1,3.7 \%)$, external jugular vein $(n=6,22.2 \%)$, retromandibular vein $(\mathrm{n}=2,7.4 \%)$, anterior jugular vein $(\mathrm{n}=1$, $3.7 \%)$, and facial vein $(n=4,14.8 \%)$. In 13 cases (48.1\%), the recipient vein was not stated. The mean flap pedicle length was $3.2 \mathrm{~cm}$. The extended pedicle length was $6.3 \mathrm{~cm}$. The mean flap ischemic time was 169.0 minutes.

In this series, the flap success rate was $100 \%$. However, postoperatively, two patients $(7.4 \%)$ required flap re-exploration due to venous thrombosis (Table 3 ). The mean postsurgical followup period was 5.6 years, with no tumor recurrence.

\section{DISCUSSION}

Ameloblastoma is a rare, benign, slowly growing, and locally in-
Table 2. Summary of fibula osteocutaneous flap characteristics

\begin{tabular}{|lcc|}
\hline Characteristics & Mean & Range \\
\hline Total fibula length & 36.1 & $32.0-42.0$ \\
Harvested fibula length & 22.6 & $19.0-27.5$ \\
Used fibula length & 13.1 & $6.5-22.0$ \\
Skin paddle length & 16.0 & $12.0-21.0$ \\
Skin paddle width & 5.4 & $3.0-8.0$ \\
\hline All unit of data is centimeter. & & \\
\hline
\end{tabular}

Table 3. Complications after reconstruction with free fibula osteocutaneous flap

\begin{tabular}{|lc|}
\hline Complications & No. (\%) \\
\hline Venous congestion & $2(7.4)$ \\
Recipient site hematoma & $1(3.7)$ \\
Recipient site infection & $2(3.7)$ \\
Flap donor site infection & $2(7.4)$ \\
Flap donor site hematoma (after primary closure) & $1(3.7)$ \\
Big toe drop & $3(11.1)$ \\
\hline
\end{tabular}

vasive odontogenic neoplasm, first described by Cusack in 1827, that frequently involves the mandible (80\%) [2]. In most cases, symptoms of the disease in its early stage are subtle, and sometimes it may only be discovered as an incidental finding during a routine radiographic examination until the neoplasm has grown into a considerable size. If left untreated, it can cause swelling of the lower jaw and displacement of teeth, causing difficulty in chewing food and even airway compromise $[2,7]$. In later stages, patients may present with tumor necrosis and infection. In this series, patients generally presented with swelling and bony deformity of the mandible associated with pain caused by the expanding neoplasm. Due to logistical issues and the tendency to seek traditional treatment first, patients came to seek medical advice only when the neoplasm had grown to a considerable size, with difficulty in mastication and speech.

The mean age of patients in this study was 29.7 years, which is younger than the ages of 39.9 years in Caucasians and 41.2 years in Asians reported by Reichart et al. [8]. Another recent study by Ruslin et al. [9] in Indonesia showed a mean age of 39.7 years. Although ameloblastoma can develop due to non-specific trauma to the tooth and periodontal structures, nutritional deficiencies, and viral infections, the exact etiology remains unknown. The relatively small sample size of this study may have contributed to the discrepancy between the results of this study and those of other studies [8-10].

Ameloblastomas can be divided into four subtypes: solid/ multicystic (91\%), unicystic (6\%), extra-osseous (2\%), and desmoplastic (1\%). The solid/multicystic type is considered to be the most aggressive type due to its high rate of recurrence if 
improperly treated. In this series, 24 patients had the solid/multicystic type, while three patients had unicystic ameloblastoma.

Conservative treatments of ameloblastoma include enucleation and cauterization of the tumor, curettage, cryotherapy, and marsupialization, all of which preserve more tissue and minimize facial deformities compared to a radical approach. However, these strategies are prone to a recurrence rate as high as $90 \%$. Another histopathologic study showed tumor extension 2-8 $\mathrm{mm}$ beyond its radiographic margins. Therefore, many authors have suggested a surgical margin of $1-2 \mathrm{~cm}$ to reduce the risk of local recurrence $[1,2,7,8,11-14]$. We employed a more radical approach by performing an en bloc resection with a bone margin of $2 \mathrm{~cm}$ to diminish the chances of recurrence. In cases with large tumors, hemimandibulectomy was performed.

Ameloblastoma is a lifelong disease, with tumor recurrence frequently occurring within 5 years of the initial diagnosis. Late recurrence occurred in 23\% of patients [14]. During our average postoperative follow-up of 5.6 years, no cases of tumor recurrence were observed.

Due to our radical approach, immediate mandibular reconstruction is vital to restore the continuity and integrity of the mandible to its preoperative state and to ensure healing of the operative site. This includes restoring the platform for the attachment of muscles involved in mastication, establishing a stable base for later dental prostheses, and providing a normal profile of the lower third of the face $[3,15]$.

The use of vascularized and non-vascularized bone has been reported by many authors in attempts to bridge mandibular defects. We favor the use of vascularized bone in the free fibula osteocutaneous flap due to its many advantages over other sources of vascularized bone. It provides the greatest length of reliable cortical bone that resists pressure and torsion, and is adequate to support osseointegrated implants [16-18]. We were able to harvest an average of $22.6 \mathrm{~cm}$ of fibula bone for reconstruction. The reliable, thin, and pliable skin paddle based on a septocutaneous blood supply enables the reconstruction of adjacent skin and mucosal defects. Conveniently sized blood vessels ease the process of performing anastomoses. The position of the donor site also allows a two-team approach during surgery, which significantly decreases the operating time. Blood loss during flap harvest is minimal $[4,19]$.

Its excellent periosteal supply allows osteotomy to be done at various locations throughout the bone to facilitate contouring and bridging of the mandibular defect. Solid union and healing were observed as early as 4-6 weeks after transfer due to its good perfusion $[4,19]$. These properties are important in oromandibular reconstruction, as complications can have lethal consequences. An unreliable skin paddle can result in a breach of the oral cavity, causing oral secretions to leak into the neck compartment, causing infection and possible carotid blowout [18]. Our technique produced a reliable skin paddle, as confirmed by the absence of cases of skin paddle loss in this study.

The vascularized fibula flap also contains more viable osteocytes, has less resorption, and is able to maintain the bone mass for later dental prostheses and rehabilitation [20,21]. Two of our patients received a dental prosthesis 1 year after surgery. The rest of the patients preferred simple dentures due to financial constraints.

Other sources of bone flaps include the scapular osteocutaneous flap, the radial forearm osteofasciocutaneous flap, and the deep circumflex iliac artery (DCIA) bone flap. They have a limited length for large bony defect reconstruction. A two-team approach is also not possible for the scapular bone flap, which results in a prolonged operating time that may cause undesirable perioperative complications. The DCIA bone flap can lead to severe donor site morbidity, such as pelvic fracture and an elevated risk of hernia and peritonitis. Pain during walking can result in a slow return to ambulation. Gait impairment can occur in some cases $[16,18,19]$.

We do not recommend non-vascularized bone grafting in mandibular defects measuring more than $4 \mathrm{~cm}$ due to its higher rate of resorption and failure [11]. Non-vascularized bone grafts are also unable to provide coverage for adjacent soft tissue defects. Foster et al. [21] reported the failure rates of non-vascularized bone grafting in mandibular reconstruction to be as high as $50 \%$ and the overall complication rate to be near $70 \%$. These success and complication rates were further influenced by adjuvant radiotherapy.

Only two of the 27 patients agreed to receive dental implants due to financial and logistic issues. The rest of the patients initially relied on dentures, but the dentures were later abandoned because they did not fit well on the reconstructed mandible and were prone to being dislodged. The patients decided to use the healthy side for chewing food. Thus, we were unable to compare the impact of the discrepancy in bone height between the reconstructed mandible and the healthy side on occlusal function. This lacuna warrants further studies with a larger sample size to look into these issues.

In conclusion, mandibular ameloblastoma should be treated with segmental mandibulectomy with a surgical margin of $2 \mathrm{~cm}$ from its radiographic margin to reduce the risk of recurrence. Mandibular defects larger than $4 \mathrm{~cm}$ should be immediately reconstructed with a free fibula osteocutaneous flap to restore mandibular function and to cover any adjacent soft tissue defect. Postsurgical follow-up should be done at least annually, as there is a lifelong risk of recurrence. 


\section{NOTES}

\section{Conflict of interest}

No potential conflict of interest relevant to this article was reported.

\section{Ethical approval}

The study was approved by the Institutional Review Board of Hospital Universiti Sains Malaysia (IRB No. USM/JEPeM/ 18100480) and performed in accordance with the principles of the Declaration of Helsinki. Written informed consents were obtained.

\section{Patient consent}

The patients provided written informed consent for the publication and the use of their images.

\section{Author contribution}

Conceptualization: Chai KS, Mat Saad AZ, Omar FH. Data curation: Chai KS, Omar FH. Formal analysis: Chai KS, Omar FH. Methodology: Chai KS, Mat Saad AZ, Omar FH. Project administration: Mat Saad AZ, Wan Sulaiman WA, Halim AS. Visualization: Chai KS. Writing - original draft: Chai KS. Writing - review \& editing: Mat Saad AZ, Wan Sulaiman WA, Halim AS. Approval of final manuscript: all authors.

\section{ORCID}

Koh Siang Chai https://orcid.org/0000-0001-9787-4510 Farah Hany Omar https://orcid.org/0000-0002-5926-2094

Arman Zaharil Mat Saad https://orcid.org/0000-0002-40036783

Wan Azman Wan Sulaiman https://orcid.org/0000-00020600-9765

Ahmad Sukari Halim https://orcid.org/0000-0001-89996403

\section{REFERENCES}

1. Effiom OA, Ogundana OM, Akinshipo AO, et al. Ameloblastoma: current etiopathological concepts and management. Oral Dis 2018;24:307-16.

2. McClary AC, West RB, McClary AC, et al. Ameloblastoma: a clinical review and trends in management. Eur Arch Otorhinolaryngol 2016;273:1649-61.

3. Wang YY, Zhang HQ, Fan S, et al. Mandibular reconstruction with the vascularized fibula flap: comparison of virtual planning surgery and conventional surgery. Int J Oral Maxillofac Surg 2016;45:1400-5.
4. Gbara A, Darwich K, Li L, et al. Long-term results of jaw reconstruction with microsurgical fibula grafts and dental implants. J Oral Maxillofac Surg 2007;65:1005-9.

5. Brown JS, Lowe D, Kanatas A, et al. Mandibular reconstruction with vascularised bone flaps: a systematic review over 25 years. Br J Oral Maxillofac Surg 2017;55:113-26.

6. Jewer DD, Boyd JB, Manktelow RT, et al. Orofacial and mandibular reconstruction with the iliac crest free flap: a review of 60 cases and a new method of classification. Plast Reconstr Surg 1989;84:391-403.

7. Hariram, Mohammad S, Malkunje LR, et al. Ameloblastoma of the anterior mandible. Natl J Maxillofac Surg 2014;5:4750 .

8. Reichart PA, Philipsen HP, Sonner S. Ameloblastoma: biological profile of 3677 cases. Eur J Cancer B Oral Oncol 1995; 31B:86-99.

9. Ruslin M, Hendra FN, Vojdani A, et al. The epidemiology, treatment, and complication of ameloblastoma in East-Indonesia: 6 years retrospective study. Med Oral Patol Oral Cir Bucal 2018;23:e54-8.

10. Gopal KS, Swathi T, Mahesh P. Ameloblastoma: a case report with a systematic review on pathogenesis and recent trends in radiographic assessment. Sch J App Med Sci 2017;5:1396-402.

11. Chana JS, Chang YM, Wei FC, et al. Segmental mandibulectomy and immediate free fibula osteoseptocutaneous flap reconstruction with endosteal implants: an ideal treatment method for mandibular ameloblastoma. Plast Reconstr Surg 2004;113:80-7.

12. Hong J, Yun PY, Chung IH, et al. Long-term follow up on recurrence of 305 ameloblastoma cases. Int J Oral Maxillofac Surg 2007;36:283-8.

13. Kaneko T, Nakamura S, Kawano R, et al. Peripheral ameloblastoma of the mandible: a case report. J Oral Maxillofac Surg Med Pathol 2016;28:565-8.

14. Milman T, Ying GS, Pan W, et al. Ameloblastoma: 25 year experience at a single institution. Head Neck Pathol 2016; 10:513-20.

15. Rommel N, Kesting MR, Rohleder NH, et al. Mandible reconstruction with free fibula flaps: outcome of a cost-effective individual planning concept compared with virtual surgical planning. J Craniomaxillofac Surg 2017;45:1246-50.

16. Lopez-Arcas JM, Arias J, Del Castillo JL, et al. The fibula osteomyocutaneous flap for mandible reconstruction: a 15year experience. J Oral Maxillofac Surg 2010;68:2377-84.

17. Peled M, El-Naaj IA, Lipin Y, et al. The use of free fibular flap for functional mandibular reconstruction. J Oral Maxillofac Surg 2005;63:220-4. 
18. Shpitzer T, Neligan PC, Gullane PJ, et al. The free iliac crest and fibula flaps in vascularized oromandibular reconstruction: comparison and long-term evaluation. Head Neck 1999;21:639-47.

19. Munoz Guerra MF, Gias LN, Rodriguez Campo FJ, et al. Vascularized free fibular flap for mandibular reconstruction: a report of 26 cases. J Oral Maxillofac Surg 2001;59:140-4.

20. Disa JJ, Hidalgo DA, Cordeiro PG, et al. Evaluation of bone height in osseous free flap mandible reconstruction: an indirect measure of bone mass. Plast Reconstr Surg 1999;103: 1371-7.

21. Foster RD, Anthony JP, Sharma A, et al. Vascularized bone flaps versus nonvascularized bone grafts for mandibular reconstruction: an outcome analysis of primary bony union and endosseous implant success. Head Neck 1999;21:6671. 